\title{
1-Acyl-Sn-Glycerol-3-Phosphate Acyltransferase Epsilon
}

National Cancer Institute

\section{Source}

National Cancer Institute. 1-Acyl-Sn-Glycerol-3-Phosphate Acyltransferase Epsilon. NCI

Thesaurus. Code C114690.

1-acyl-sn-glycerol-3-phosphate acyltransferase epsilon (364 aa, $\sim 42 \mathrm{kDa}$ ) is encoded by the human AGPAT 5 gene. This protein plays a role in the synthesis of phosphatidic acid from lysophosphatidic acid. 